\title{
Introduction to the Special Issue: Combinatorics and Geometry
}

\author{
Evangelos Kranakis
}

Published online: 20 February 2015

(C) Springer Japan 2015

This special issue is an outgrowth of the Mexican Conference on Combinatorics and Geometry held in Oaxaca, Mexico, on November 11-15, 2013 to celebrate Jorge Urrutia's 60th birthday.

Jorge is one of those rare individuals endowed with a wonderful mathematical sense of creativity. All he needs is a pencil and paper but is always willing to help with a proof, always available to interact anytime and anywhere, always tireless to elaborate on a new mathematical paradigm, and always delighted and excited when a new idea leads to a beautiful new theorem. For many of us Jorge has been not only a teacher, a friend, and a research collaborator but also an inspirational figure whose love for mathematics has been a model for making the subject approachable and lively and for contributing to a deeper understanding of its beautiful, undiscovered secrets. As Isaac Newton said "I keep the subject constantly before me and wait till the first dawnings open little by little into full light".

Most of the papers included in this issue were presented at the conference and were all rigorously refereed upon submission to this special issue. They include a variety of interesting topics reflecting the varied interests of the participants: Reversibility of convex polygons, Trapping disks by four lines, Extremal antipodal polygons, Monitoring the Plane with Rotating Radars, Geometric biplane graphs, Geometric Spanning Cycles in Bichromatic Point Sets, Cross-Intersecting Families of Vectors, Empty Triangles in Good Drawings of the Complete Graph, and Isoperimetric enclosures. We hope you will enjoy reading and learn from them as much beautiful mathematics as we did.

\footnotetext{
E. Kranakis $(\varangle)$

Ottawa, Canada

e-mail: kranakis@scs.carleton.ca
} 
We would also like to take this opportunity to thank all the anonymous referees for their meticulous refereeing and detailed comments they provided to all the authors, as well as the editors of the journal for their patience and understanding.

Ruy Fabila

Evangelos Kranakis

Pedro Ramos

February, 2015 